\author{
L. Hakkaart-van Roijen \\ B.W.C. Zwirs \\ C. Bouwmans \\ S.S. Tan \\ T.W.J. Schulpen \\ L. Vlasveld \\ J.K. Buitelaar
}

\section{Societal costs and quality of life of children suffering from attention deficient hyperactivity disorder (ADHD)}

Accepted: 21 December 2006

Published online: 4 May 2007
L. Hakkaart-van Roijen $(\bowtie)$

C. Bouwmans $\cdot$ S.S. Tan

Institute for Medical Technology

Assessment (iMTA)

Erasmus MC

P.O. Box 1738

3000 Rotterdam DR, The Netherlands

Tel.: +31-10/4088567

Fax: +31-10/4089081

E-Mail: 1.hakkaart-vanroijen@ erasmusmc.nl

B.W.C. Zwirs - T.W.J. Schulpen University Medical Centre Utrecht Utrecht, The Netherlands

L. Vlasveld

Reinier de Graaf Group

Delft, The Netherlands

¿̊. J.K. Buitelaar

University Medical Centre St. Radboud

Nijmegen, The Netherlands
Abstract Background The impact of attention deficit hyperactive disorder (ADHD) in the Netherlands on health care utilisation, costs and quality of life of these children, as well as of their parents is unknown. Objective The aim of this study was to assess the direct medical costs of patients suffering from ADHD and their quality of life as well as the direct medical costs of their mothers. Study design We selected a group of 70 children who were being treated by a paediatrician for ADHD based on the DSM-IV diagnostic criteria for ADHD. For comparison's sake, we also included a non-matched group of 35 children with behaviour problems and 60 children with no behaviour problem from a large school population-based study on the detection of ADHD. We collected information on the health care utilisation of the children applying the Trimbos and iMTA questionnaire on Costs associated with Psychiatric illness' (TiC-P). Their health related quality of life was collected by using the Dutch 50 item parent version of the Child Health Questionnaire (CHQ PF50). Measurements were at baseline and at 6 months. Subsequently, we collected data on the health utilisation of the mothers and their production losses due to absence from work and reduced efficiency. Results The mean direct medical costs per ADHD patient per year were $€ 2040$ or $€ 1173$ when leaving out one patient with a long-term hospital admission, compared to $€ 288$ for the group of children with behaviour problems and $€ 177$ for the group of children with no behaviour problems. The direct medical costs for children who had psychiatric co-morbidities were significantly higher compared to children with ADHD alone. The mean medical costs per year for the mothers of the ADHD patients were significantly higher than for the mothers of the children with behaviour problems and the mothers of children with no behaviour problems respectively $€ 728$, $€ 202$ and $€ 154$. The physical summary score showed no significant differences between the groups. However, the score on the Psychosocial Summary Score dimension was significantly lower for ADHD patients compared to the scores of the children in the two other samples. The mean annual indirect costs due to absence from work and reduced efficiency at work were $€ 2243$ for the mothers of the ADHD patients compared to $€ 408$ for the mothers 
of children with behaviour problems and $€ 674$ for the mothers of children with no behaviour problems. Conclusion Our study showed that the direct medical costs of ADHD patients were relatively high. Additionally, our study indicated that ADHD appears to be accompanied by higher (mental) health care costs for the mothers of ADHD patients and by increased indirect costs for this group.

Key words ADHD societal costs - quality of life

\section{Introduction}

Attention deficit hyperactivity disorder (ADHD) is characterised by a persistent pattern of overactivity, inattention and impulsivity that is pervasive across situations and accompanied by substantial impairments in family and social relationships [1]. ADHD is a relatively common disorder that occurs in $8 \%$ of the children between five and fourteen years old [6]. The prevalence rate among boys is higher than for girls [22]. Typical symptoms of children suffering from ADHD are failing to remain seated, talking excessively, playing noisily, and blurting out answers before the questions have been completed [1]. The symptoms of ADHD may interfere with a person's ability to conduct normal daily activities. ADHD is associated with broad impairment in many Health Related Quality of Life (HRQoL) parameters, including academic performance, behaviour at school, peer relations and family function [5]. Studies have indicated that, compared to their peers without ADHD, young people with ADHD also exhibit higher levels of comorbidity with other psychiatric disorders as oppositional defiant disorder, conduct disorder, anxiety disorder and depression, and with somatic conditions as asthma [2]. Furthermore, they have an increased risk of accidents and severe injury [2]. ADHD may also affect the health and ability to work of family members [2]. The coexisting psychiatric conditions may add to the impairment associated with ADHD, as well as to the disorder's economic burden.

Because of the broad impact of ADHD, the disorder is likely to have significant economic implications for society and for the quality of life of patients and their family. Studies have shown that subjects with ADHD have a substantially greater use of care, next to an impaired quality of life $[7,15]$. A recent paper by Matza, reviewing and summarizing the growing body of literature on the economic burden of ADHD primarily published in the United States [8] clearly showed that ADHD places a substantial economic burden on patients, families and third-party players [8]. Results of medical costs studies showed that children with ADHD had higher annual medical costs than either matched controls (difference ranged from $\$ 503$ to $\$ 1343$ ) or non-matched controls (difference range from \$207 to \$1560) without ADHD [8]. A limited number of studies have examined other economic implications of ADHD, such as the cost to families, indirect costs associated with work loss among parents of children suffering from ADHD [16, $25,26]$.

A number of studies have also indicated that the impact of ADHD on HRQoL is significant [11, 19, 27]. Escobar et al. showed that ADHD interferes to an even greater extent than asthma with the daily lives of children, parents and families, primarily in areas related to psychosocial functioning [5].

The impact of the disorder in the Netherlands on health care utilisation, costs and quality of life of these children, as well as of their parents is unknown. Hence, the aim of this study was to estimate the direct costs incurred for the medical care of patients with ADHD. Furthermore, we assessed the HRQoL of these patients by using the Child Health Questionnaire [13, 27]. Additionally, we estimated the direct medical costs for care provided to their mothers and the indirect costs due to absenteeism, reduced efficiency at work and impediments due to behaviour problems of their child. We compared these results with estimates for a group of children with behaviour problems and with a group of non-matched controls. Additionally, we compared the costs and quality of life scores within the group of ADHD patients with and without psychiatric comorbidity.

\section{Methods}

\section{Study sample}

For this study, we selected a group of 70 children who were being treated by a paediatrician for ADHD. ADHD patients were diagnosed by a multidisciplinary team of medical doctors, children's psychologist and an orthopedagogue. The diagnosis was based on the DSM-IV diagnostic criteria for ADHD. This group will 
be referred to as 'ADHD patients' throughout the paper. For comparison's sake, we also recruited two groups of children from a study on the detection of ADHD among children of different ethnic origin in the Netherlands (ADEON) [28]. The aim of the ADEON project was to develop and validate a tool (diagnostic score) that could be used as a rapid initial assessment of the probability of ADHD in non-referred school age children, taking ethnicity into account. Initially, teachers in Amsterdam and Utrecht were asked to rate a population of 3,000 six to seven year-olds, made up of the pupils in their classes, on a rating scale. Some 300 children (150 with low scores and 150 with high scores for externalizing items) were subsequently invited for detailed assessments. We selected a group of 60 children from the ADEON study population with low scores, i.e. no behaviour problems, and 35 children with behaviour problems, i.e. with scores in the 90th percentile and higher. Of the latter group, a total of 12 children were eventually diagnosed with ADHD by a psychiatrist, based on the DSM-IV diagnostic criteria for ADHD.

Unfortunately, we could not match the three groups by age and sex, as the group of ADHD patients being treated by a paediatrician was relatively old and contained relatively more boys than the group of six to seven year-olds recruited from the ADEON study. In the paper, the group of children with low scores will be referred to as children with 'no behaviour problems' and the group with high scores as children with 'behaviour problems'.

\section{Measures and data analyses}

Our study was conducted from a societal perspective and included assessments of quality of life, direct medical and direct medical- and indirect costs of the mothers. Information on the health care utilisation of the children and their health related quality of life were collected using questionnaires, which were sent to the home address of the participants. Enrolment commenced in November 2002 and stopped in February 2004; follow-up data were collected until August 2004. For all groups measurements were at baseline and at 6 months using a postal questionnaire. The recall period was 3 months; we assumed this period to be representative for the 6-month period. Subsequently, we collected data on the health utilisation of the mothers and their production losses due to absence from work and reduced efficiency. In cases where the mother was unable to fill out the questionnaire, we asked another person to do so. These subjects were also asked to indicate their relationship to the child. For the purpose of brevity, the term 'mother' should be assumed to include both the mothers and the small group of respondents with some other relationship to the child.

The collected data included socio-demographic characteristics of the children and their mothers, such as sex, age, educational level of the children, and the work status of the mother at baseline. For the group of ADHD patients, we collected data on psychiatric comorbidity from the patient files. Comorbidity was defined as the presence of any additional or coexisting psychiatric disorder stated in the relevant patient's file. Also, we compared the baseline scores with the scores at six months after of incident patients were included in the ADHD sample. An incident case was defined as a respondent whose first contact with the paediatrician occurred on the date of inclusion, e.g., at the baseline measurement. Where data from the second measurement, i.e., at six months, were missing, we applied linear extrapolation (LE). Applying LE, the direct medical costs and indirect costs due to absenteeism and reduced efficiency at work were extrapolated to one year by multiplying the costs of first six months by two.

\section{Direct and indirect costs}

Direct costs were defined as the monetary valuation of the resources used to detect and treat medical problems. The indirect costs were defined as the productivity lost due to absenteeism and reduced efficiency at work.

For all groups we used the 'Trimbos and iMTA questionnaire on Costs associated with Psychiatric illness' (TiC-P) to collect data on direct and indirect costs [8]. The first part of the TiC-P consists of questions on the number of contacts with health care providers, including the general practitioner (GP), psychiatrist, medical specialists, speech therapist, (psychiatric) day care/hospital length of stay, medication and out-of-pocket costs. Bottom-up methodology was used to calculate the total direct medical costs; that is, the total number of medical contacts (outpatient visits, hospital length of stay, use of medication, etc.) was multiplied by the 2004 reference unit prices of the corresponding health care service [21]. In this way, we assessed the overall direct medical costs of this group of patients suffering from ADHD.

Next, we assessed health-related school absenteeism and failure to participate in sports, without, however, assigning a monetary value to these items. The recall period for the TiC-P was three months. In estimating the costs over the period of half a year, we assumed that the costs per three months could be taken as representative of the preceding six months. The annual costs were calculated by adding up the costs per half year. 
In parallel, the mothers were asked to fill out the TiC-P with regard to their own health care utilisation. The TiC-P for mothers also included a short form of the Health and Labour questionnaire (HLQ) for collecting data on productivity losses [24]. The ShortForm HLQ (SF-HLQ) consists of three modules that measure productivity losses: absence from work, reduced efficiency at work, and difficulties with job performance [3]. The days of absence from work and actual cost of hours missed at work due to healthrelated problems were valued by the net income per day and per hour, respectively. The friction-cost method was applied to assess the productivity losses [12]. This method takes into account the economic circumstances that limit the losses of productivity to society, which is related to the fact that a formerly unemployed person may replace a person who becomes disabled [12]. The period needed to replace a worker (the so-called friction period) is estimated to be 154 days. The number of lost working days per respondent was calculated taking into account the number days and hours of paid employment per week.

To collect data on reduced efficiency at work, respondents with paid jobs were asked to estimate the number of extra hours they would have had to have worked to compensate for the health-related work productivity loss incurred. We estimated an impediment score to assess the amount of difficulty experienced in performing both paid and unpaid jobs. Unpaid jobs were defined as household work, shopping, childcare (in this context, taking care of one's own children), and odd jobs around the house.

Individuals with paid jobs were asked to indicate the degree of impediment they experienced while performing their paid jobs. The response categories were: $0=$ 'no impediment', $1=$ 'some impediment', and $2=$ 'a lot of impediment'. The impediment score for a paid job ranged from 0 to 2 .

Moreover, a descriptive instrument comprising seven items was used to evaluate underlying problems causing reduced efficiency while performing a paid job, and to calculate the efficiency score. The items were concentration, working pace, need to be alone, decision making, postponement of work, taking over work by others and other (the latter was an open-end question). Response modalities were $1=$ 'never', $2=$ 'sometimes', $3=$ 'often', and $4=$ 'always'. The efficiency score for a paid job was calculated by adding up the scores for the first six items and ranged from 6 to 24 .

For each category of a unpaid work (household work, shopping, childcare, odd jobs around the house), the response categories and scores were comparable with those for a paid job: $0=$ "no impediment', $1=$ 'some impediment' and $2=$ 'a lot of impediment'. The impediment score for unpaid work was calculated by adding up the scores for four categories of unpaid work; the scores ranged from 0 to 8 .

\section{Quality of life}

The quality of life of the children was assessed by using the Dutch 50 -item parent version of the CHQ [27]. The CHQ-PF50 is a generic health status instrument designed to measure the physical and psychosocial well being of children aged five and older. The CHQ-PF50 assesses children's physical and mental health and the parent's perception of the extent to which problems in these areas interfere with peer and school activities, family activities, and the lives of the parents. It has been applied in general populations and as well in studies with chronically ill children in several countries [14]. The CHQ-PF50 includes a broad spectrum of child and family focused health areas, broken down into: physical functioning, role/social limitations-emotional/behavioural, role / social limitations physical, bodily pain/discomfort, behaviour, mental health, self-esteem, general health perceptions parent impact-emotional, parent impact time, family activities and family cohesion. Most items require parents to respond using a four week recall period, and items are rated on 4-point, 5-point, and 6-point Likert scales. Two summary scores, the Psychosocial Summary Scale and the Physical Summary Scale, were derived for the weighted combinations of the domain subscale scores. The summary scores were transformed to achieve a score range of 0-100, with higher scores representing a better HRQoL. The CHQ-PF50 has been tested and validated with normative values developed for ADHD, asthma, cystic fibrosis, epilepsy and juvenile rheumatoid arthritis for the US population [14].

\section{Data analysis and statistics}

Statistical analysis was performed using the SPSS statistical software (version 13.0; SPPS Inc., Chicago, IL, USA). We applied non parametric tests to assess differences between groups and differences in baseline and follow-up scores using a significance level of $P<0.05$.

\section{Results}

\section{Study sample}

The response rate of the group of ADHD children treated by a paediatrician was $92 \%(n=65)$. For the group of children with behaviour problems, the response rate was $94 \%(n=33)$, including 12 respon- 
Table 1 General characteristics of the three study groups, children with ADHD (DSM-IV) treated by a pediatrician, children with behaviour problems and children with no behaviour problems

\begin{tabular}{|c|c|c|c|}
\hline Characteristics & $\begin{array}{l}\text { ADHD } \\
(n=65)\end{array}$ & $\begin{array}{l}\text { Behaviour problems } \\
(n=33)\end{array}$ & $\begin{array}{l}\text { No behaviour problems } \\
(n=47)\end{array}$ \\
\hline Average age child, years; Mean (SD) & $10.5(2.7)$ & $9.3(1.0)$ & $7.8(1.0)$ \\
\hline Men child; $N(\%)$ & $52(80 \%)$ & $17(52 \%)$ & $17(36 \%)$ \\
\hline \multicolumn{4}{|l|}{ Comorbidity child; $N(\%)$} \\
\hline Oppositional defiant disorder & $5(8 \%)$ & na & na \\
\hline Anxiety disorder & $1(2 \%)$ & na & na \\
\hline Pervasive development disorder & $6(9 \%)$ & na & na \\
\hline Other psychiatric not specified disorders & $3(5 \%)$ & na & na \\
\hline \multicolumn{4}{|l|}{ Type of school; $N(\%)$} \\
\hline Elementary school & $43(66 \%)$ & $33(100 \%)$ & $47(100 \%)$ \\
\hline Secondary school & $12(18 \%)$ & $0(0 \%)$ & $0(0 \%)$ \\
\hline Special education & $10(15 \%)$ & $0(0 \%)$ & $0(0 \%)$ \\
\hline Average age mother, years; Mean (SD) & $38.6(4.6)$ & $37.5(7.7)$ & $38.0(5.4)$ \\
\hline Mothers* with paid work; $N(\%)$ & $50(77 \%)$ & $18(55 \%)$ & $27(57 \%)$ \\
\hline
\end{tabular}

$\mathrm{ADHD}=$ attention-deficit hyperactivity disorder

dents diagnosed with ADHD according to the standard DSM-IV criteria. The response rate for the group of children with no behaviour problems was relatively low, $78 \%(n=47)$.

Table 1 presents the general characteristics at baseline of all the respondents in the three groups. The mean age of the patients in the ADHD group was higher than that of the two other groups, as was the number of boys in this group. Fourteen (22\%) ADHD patients were found to have a co-morbid psychiatric diagnosis; only one patient, however, had more than one co-morbid psychiatric condition. The co-morbidities reported included oppositional defiant disorder (ODD), anxiety disorder, pervasive development disorder (PDD) and other psychiatric not otherwise specified disorders. Fifty-one percent $(n=33)$ of the ADHD patients visited the paediatrician for the first time at baseline and were treated as incidence cases in the analyses.

Fifteen percent of the ADHD patients were in special education, compared to none in the other study groups. Nearly half of the ADHD patients had missed one or more days of school during the three months preceding the interview. At six months, the response rate was $89 \%(n=58), 79 \% \quad(n=26)$, and $91 \%$ $(n=43)$ for the ADHD patients, the group of children with behaviour problems and children with no behaviour problems, respectively. There were no between-group differences in missing service use data.

\section{Direct costs}

Table 2 presents the mean annual direct medical costs incurred by the ADHD patients and their mothers.
These costs are broken down by type of medical service and the percentage of respondents using a particular medical service. The mean direct medical costs per child per year were over $€ 2000$, compared to $€ 288$ for the group of children with behaviour problems and $€ 177$ for the comparison group.

The cost of hospital stays accounted for over one third of the annual costs for the ADHD patient group; the brunt of these costs were, however, borne by a single patient's 84 -day admission to a psychiatric hospital. If this patient is left out of the analysis, the mean direct medical costs fall to $€ 1173$. This reflects the substantial impact of a long stay admission to hospital. Ambulatory mental health care accounted for a quarter of the mean direct costs, as shown in Table 2. The paediatrician was the most commonly consulted health service. However, this was to be expected, as the group of children with ADHD was selected from a population being treated by a paediatrician for ADHD. Three quarters of the children diagnosed with ADHD were on medication, which accounted for $13 \%$ of the direct medical costs. The out-of-pocket costs per year for children with ADHD were on average nearly $€ 20$ (SD $€ 130$ ). Children of both other groups did not have out-of-pocket costs.

The mean direct medical costs per year for children who had psychiatric co-morbidities were $€ 5908$, compared to only $€ 974$ for children with ADHD alone. If the child who was admitted to hospital is left out, the mean costs of the group of patients with comorbidity would be reduced to $€ 1946$.

The mean medical costs per year for the mothers of the ADHD patients were over $€ 700$, as shown in Table 2 . The total mean annual direct medical costs for 
Table 2 Mean direct medical cost per year for children with ADHD and their mothers ${ }^{\mathrm{a}}$ (in euros)

\begin{tabular}{|c|c|c|c|}
\hline Type of medical service & $\begin{array}{l}\text { Costs per } \\
\text { year (sd) }\end{array}$ & $\begin{array}{l}\text { Percentage of the } \\
\text { total direct } \\
\text { medical costs }\end{array}$ & $\begin{array}{l}\text { Respondents } \\
\text { using the } \\
\text { service [ } n(\%)]\end{array}$ \\
\hline \multicolumn{4}{|l|}{ Children } \\
\hline General practitioner & $33(18)$ & 2 & $19(29)$ \\
\hline Ambulatory mental health care & $501(416)$ & 25 & $21(32)$ \\
\hline Psychiatric practice & $79(71)$ & 4 & $14(22)$ \\
\hline Out-patient psychiatrist & $52(90)$ & 3 & $9(14)$ \\
\hline School doctor & $25(22)$ & 1 & $8(12)$ \\
\hline Paediatrician & $144(26)$ & 7 & $55(85)$ \\
\hline Medical specialist & $26(21)$ & 1 & 7 (11) \\
\hline Physiotherapist & $30(35)$ & 1 & $3(5)$ \\
\hline Speech therapist & $75(88)$ & 4 & $6(9)$ \\
\hline Social worker & $60(91)$ & 3 & $5(8)$ \\
\hline Alternative health practitioner & $32(64)$ & 2 & $4(6)$ \\
\hline Hospital days & $723(2676)$ & 35 & $1(2)$ \\
\hline Medication & $260(59)$ & 13 & $48(75)$ \\
\hline Total & $2,040(943)$ & 100 & \\
\hline \multicolumn{4}{|l|}{ Mothers $^{\mathrm{a}}$} \\
\hline General practitioner & $54(27)$ & 7 & $26(40)$ \\
\hline Ambulatory mental health care & $412(386)$ & 57 & $19(30)$ \\
\hline Psychiatric practice & $74(82)$ & 10 & $7(11)$ \\
\hline Out-patient psychiatrist & $55(89)$ & 8 & $6(9)$ \\
\hline Company doctor & $11(19)$ & 2 & $6(9)$ \\
\hline Social worker & $57(59)$ & 8 & $8(12)$ \\
\hline Consultant alcohol \& drugs & $2(7)$ & 0 & $1(2)$ \\
\hline Alternative health practitioner & $28(39)$ & 4 & $3(5)$ \\
\hline Medication & $35(14)$ & 5 & $28(43)$ \\
\hline Total & $728(432)$ & 100 & \\
\hline
\end{tabular}

a In this study this group consisted of $82 \%$ mothers, $17 \%$ fathers, and $1 \%$ others, referred to as 'mothers' $\mathrm{ADHD}=$ attention-deficit hyperactivity disorder

the mothers of the children with behaviour problems were $€ 202$, and for the mothers of the children with no behaviour problems, $€ 154$.

For the mothers of the ADHD patients, visits to the ambulatory mental health care service accounted for over half of the mean direct medical costs per year. As a group, these mothers made significantly more use of and incurred significantly higher costs for mental health care, including the costs of ambulatory mental health care, psychiatric practice and outpatient psychiatric care, than did the mothers of the group of children with behaviour problems and of the mothers of the children with no behaviour problems, respectively $€ 541, € 47$ and $€ 35$. Furthermore, 4 out of 10 mothers of the ADHD patients had consulted the general practitioner and slightly over $40 \%$ of the mothers were using psychotropic medications. Overall, a quarter of the mothers of the ADHD patients indicated that they had used health care services for themselves for reasons related to the behaviour problems of their child.

The annual mean direct medical costs for mothers of the ADHD patients with psychiatric comorbidity were $€ 839$, compared to mean direct medical costs of $€ 755$ for mothers of children with ADHD only

\section{Indirect costs}

\section{Absence from work}

About three quarters of the mothers of the ADHD patients had a paid job (see Table 1). This percentage was higher than that of the mothers in the two other groups (Table 1). Table 3 shows the indirect cost data for mothers with a paid job. For the group of mothers of the ADHD patients, $16 \%$ were absent from work due to health-related problems. The mean number of days absent from work per year was over 17 days. Generally, the period of absenteeism was relatively short; only one mother indicated she had been absent for a period of longer than two weeks. The mean indirect costs per year due to absence from work were $€ 1691$ per mother. By comparison, the mean annual number of missed days at work was nearly three days for the mothers of the children with behaviour problems, with corresponding mean annual costs of 
Table 3 Average number of days lost due to absence from work, reduced efficiency and indirect costs per year of mothers ${ }^{\mathrm{a}}$ of ADHD patients $(n=50)$

\begin{tabular}{lcc}
\hline Type of production loss & Number of days & Costs (in euros) \\
\hline Absence from work & 17.3 & 1,691 \\
Reduced Efficiency & 5.1 & 552 \\
Total & 22.4 & 2,243 \\
\hline
\end{tabular}

${ }^{a}$ This group consisted of $82 \%$ mothers, $17 \%$ fathers, and $1 \%$ others, referred to as 'mothers'

$A D H D=$ attention-deficit hyperactivity disorder

$€ 408$, and six days for the screen negatives with the corresponding mean annual costs of $€ 674$.

\section{Reduced efficiency at work}

The mean productivity losses due to reduced efficiency at work were approximately five days per year for the mothers of the ADHD patients (Table 3). The mean annual associated costs were nearly $€ 552$. Of the group of mothers with a child with behaviour problems, only one mother indicated that she had experienced health-related productivity loss due to reduced efficiency. The mean number of days lost due to reduced efficiency was therefore low, about half a working day per year. The group of mothers of the screen negatives reported no production losses due to reduced efficiency.

\section{Difficulties with job performance}

A third of the mothers of the ADHD patients with a paid job experienced difficulties at work because of health-related problems at baseline. The impediment score associated with paid jobs was 0.38 for mothers of the ADHD patients. This score was significantly higher than the 0.18 found for mothers of children with behaviour problems and the 0.15 scored by the screen negative sample, indicating that mothers of ADHD patients indeed experience more difficulties. Approximately one third (36\%) of the mothers of the ADHD patients indicated they needed to be alone. Approximately one fifth occasionally experienced problems with concentration, working pace and decision making. Only $6 \%$ of the mothers of the ADHD patients reported having to postpone work. The resulting efficiency score was 13.0 for the mothers of the ADHD patients versus 6.31 for the mothers of children with behaviour problems and 6.44 for the mothers of the children with no behaviour problems. This suggests that mothers of ADHD patients indeed experience more problems in performing their jobs.

Twenty percent of the mothers of the ADHD patients indicated that they experienced problems in

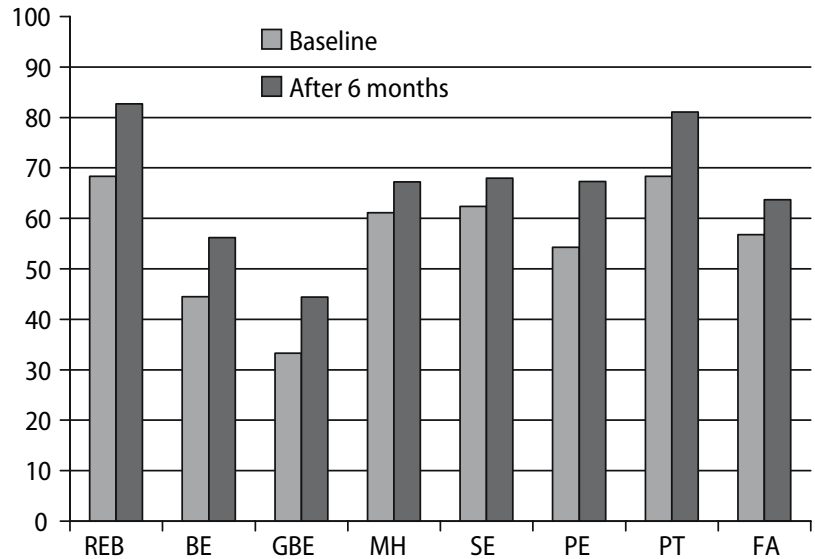

Fig. 1 Average score on the CHQ-PF 50 at baseline and 6 months of incident ADHD patients. $A D H D=$ attention-deficit hyperactivity disorder

performing unpaid work, e.g., household work. Over 12 percent of the mothers reported difficulties in taking care of their child(ren). The mean impediment score for unpaid work in the group of mothers of the ADHD patients was higher than for the mothers of the children with no behaviour problems and children with behaviour problems. However, the difference between the samples was not significant: 0.67 versus 0.47 and 0.43 respectively.

\section{Quality of life}

Table 4 presents the mean scores of these measurements for the different items of the CHQ-PF50 and the summary scores in the three groups at baseline. The physical summary score (PhS) showed no significant differences between the groups. However, the score on the Psychosocial Summary Score (PsS) dimension was significantly lower for ADHD patients compared to the scores of the children in the two other samples. Additionally, the global parent's rating of the present health of the child (GGH) and scores on general health $(\mathrm{GH})$ were significantly lower in the ADHD patients. This dimension represents the parents' perception of the health of their children in general and expectations regarding the health of their children in the future.

For the group of incident ADHD patients, the Psychosocial Summary Score was significantly higher compared with the baseline scores after 6 months of treatment (33.8 versus 41.0, respectively). Furthermore, scores for Role/Social Limitations-Emotional/ Behaviour problems (REB), Behaviour (BE), General Behaviour (GBE), Mental Health (MH), Parental Impact-Emotional (PE), Parental impact Time (PT) and Family Activities (FA) were significantly improved at 
Table 4 Mean scores on the sub dimensions and the summary scores of the CHQ-PF50 for ADHD patients, children with behaviour problems and children with no behaviour problems at baseline (higher scores indicates better functioning)

\begin{tabular}{llll}
\hline & ADHD patients & Behaviour problems & No behaviour problems \\
\hline Global Health (GGH) & 75.5 & 79.9 & 82.3 \\
Physical Functioning (PF) & 97.2 & 96.8 & 96.2 \\
Role/Social Limitations-Emotional/Behavioural (REB) & $76.9^{*}$ & 95.7 & 94.8 \\
Role/Social Limitations-Physical (RP) & 93.3 & 95.4 & 98.2 \\
Bodily Pain/Discomfort (BP) & 82.5 & 84 & 86.7 \\
Behavior (BE) & $55.1^{*}$ & 79.7 & 77.4 \\
Global Behavior Item (GBE) & $47.2^{*}$ & 71.8 & 72.3 \\
Mental Health (MH) & $63.5^{*}$ & 79.3 & 81.9 \\
Self Esteem (SE) & $66.0^{*}$ & 78.7 & 80.4 \\
General Health Perceptions (GH) & 79.8 & 82.4 \\
Parental Impact-Emotional (PE) & $63.4^{*}$ & 83.4 & \\
Parental Impact-Time (PT) & $78.3^{*}$ & 87.1 & \\
Family Activities (FA) & $64.8^{*}$ & 96.9 & \\
Family Cohesion (FC) & $57.6^{*}$ & 92.1 & 87.8 \\
Physical Summary Score (PhS) & 57.5 & 71.4 & \\
Psychosocial Summary Score (PsS) & $38.9^{*}$ & 55.8 & \\
\hline
\end{tabular}

*Significantly different

ADHD = attention-deficit hyperactivity disorder

six months, see Fig. 1. The improvement of the scores on the CHQ-PF 50 at 6 months compared to the baseline measurement was entirely due to the higher scores of the incident ADHD patients.

As is apparent from the literature, comorbidity has an additional effect on the quality of life in children with ADHD [11]. The Psychosocial Summary Score was lower for the ADHD patients with psychiatric comorbidity compared to the group of patients with ADHD only, namely 31.7 and 40.8 respectively. Differences were due to significantly worse scores in the ADHD patient group with comorbid psychiatric disorders on emotional or behavioural problems (REB), emotional behaviour (BE) and general behaviour (GBE) and self esteem (SE), compared to the ADHD patients with no psychiatric comorbidity. We also found significantly lower scores on the parents' time (PT) for this group.

\section{Conclusion}

The purpose of this study was to estimate the health care utilisation, direct medical costs and quality of life of ADHD patients. To this end, we compared the overall direct medical costs and quality of life score with a non-matched group of children with behaviour problems and with a group of children with no behaviour problems (screen negatives). Additionally, we assessed the health care utilisation and direct medical costs of the mothers and their indirect costs due to production losses at work. The response rate was high (92\%), as was the percentage of boys in the group of ADHD patients (80\%). ADHD seems to impair school performance, as $15 \%$ of the ADHD patients attended special education. The annual direct medical costs of the ADHD patients were estimated at $€ 2040$. These costs were seven to eleven times higher than the direct medical costs of children with behaviour problems and of the children with no behaviour problems, respectively. Psychiatric hospital days and ambulatory mental health care accounted for over half of the costs. Medication accounted for $13 \%$ of the costs. However, direct medical costs were highly skewed due to one patient with a long term hospital admission. Leaving out this patient the direct medical costs of the ADHD patients were on average $€ 1173$. ADHD patients with psychiatric comorbidity had higher health care costs than the ADHD patients with no psychiatric comorbidity.

The mean annual direct costs of the mothers of the ADHD patients were $€ 728$. The total indirect costs due to absence from work and reduced efficiency at work were $€ 2243$. Furthermore, mothers of ADHD patients had higher impediment scores associated with a worse performance in paid and household activities. Direct medical costs and indirect costs for the mothers of ADHD patients were substantially higher than for the mothers of the other two groups. Comorbidity of the child seemed not to influence the costs of the mother.

The quality of life of the ADHD patients was significantly worse in the psychosocial domain. However, ADHD appeared not to have an impact on the physical domain. Furthermore, ADHD had a negative impact on the parental time and family activities. 


\section{Discussion}

This is the first cost-of-illness study of ADHD conducted in the Netherlands. In line with similar studies carried out elsewhere, our study showed that the direct medical costs of ADHD patients were relatively high. Additionally, our study indicated that ADHD appears to be accompanied by higher (mental) health care costs for the mothers of ADHD patients and by increased indirect costs for this group. This would seem to concur with the presented relative negative scores on parental time and family activities on the CHQ-PF50. HQoL assessment consistently showed a considerable impairment in the psychological and social functioning of the children suffering from ADHD.

The group of children with a high score on a screenings list for behaviour problems were, by contrast, not found to have increased direct medical costs and worse HRQoL. Our study showed that the percentage of mothers with a paid job was higher in the group of ADHD patients. There was no difference in mean age and marital status between the groups which may have explained the variance in work status of the mothers. Further research on this subject is needed. Our study had several limitations. Our data pertained to only a small group of ADHD patients, who were being treated by a paediatrician. This group may not be considered to be representative of ADHD patients in the Netherlands. The ADHD patients were identified on the bases of a dependent variable; in treatment for ADHD by a paediatrician. This may introduce an upward bias to the costs. According to the paediatrician, this group of ADHD patients should be regarded as a group of patients with moderate ADHD. Hence, mild and severe ADHD patients were probably underrepresented in this sample. However, unfortunately we did not have data on severity. Furthermore, the small sample limits the external validity. The number of ADHD patients with psychiatric comorbidity was small compared to other studies [7]. The reason for this could be twofold. Firstly, the paediatrician may not have been consistent in recording comorbidity. Secondly, the ADHD patients who were treated by a paediatrician may well constitute a group with less severe ADHD. Moreover, due to the study design, we were unable to match the two other study groups on age and sex. Hence, the comparison of the results is limited; it only offered some indications of differences between ADHD patients and two other non-matched groups of children.

In the group of children with behaviour problems, the number of patients with ADHD according to the criteria of the DSM-IV was too small to explore meaningful subgroup analyses.
Compared to a recent Cost-of-illness study for Belgium our direct medical cost estimate was relatively high. This may be partly due to the variance in patient selection, the parents were members of the Flemish-speaking ADHD society [4] and may not be all under treatment for ADHD during the study. Furthermore, the costs of hospitalisation and mental health care in our study were relatively high. In our study the costs of hospitalisation were high due to an admission of 84 days to a psychiatric hospital of one patient.

Another limitation of the study was that questions regarding use of medical resources and productivity losses were not specific to ADHD, but referred to health problems in general. Hence, the cost estimate in this study referred to the costs of direct medical costs of patients suffering from ADHD and not to the costs of ADHD for these patients. Due to the symptoms of ADHD, parents of ADHD patients cannot easily distinguish between problems related to ADHD itself and other mental and psychosomatic health problems. On the one hand, this may lead to the costs of ADHD being overestimated. On the other hand, our study failed to include severely ill ADHD patients, leading to an underestimate of the mean costs.

The annual costs were based on two measurement points with a recall period of three months. Studies have indicated that the accuracy of data on health care utilisation decreases with long recall periods $[9,10$, 17]. We assumed that the three months were representative for the entire six-month period.

In case of missing data at the second measurement moment (at six months) we applied a naïve method to deal with missing observations compared to, for instance, multiple imputation [20]. However, the influence of the imputing method was limited as the number of missings was small.

The number of children with ADHD attending special education was significantly higher. We did not value these costs to society. However, these costs are probably relatively high compared to other costs components.

We collected no data on the cost of crime. International studies have shown that ADHD patients are at an increased risk for criminal activities [18]. However, these costs were expected to be low in the age group included in our study.

The scores of the children with no behaviour problems on the CHQ-PF50 were comparable to the scores obtained in a group of Dutch school-aged children, indicating the comparison group may be used as a reference group [23].

In our study, the group of ADHD patients visiting the paediatrician for the first time at baseline showed better HQoL after six months, suggesting that the 
treatment of ADHD was effective. However, this result does not assess the marginal cost-effectiveness of individual treatments of ADHD.

A cost-of illness estimate cannot be interpreted as the savings to be achieved with new successful medical interventions. However, the results of a cost-of illness study may provide helpful information when designing a cost-effectiveness study. For instance, the estimates of the direct medical costs in certain patient groups may be useful in estimating the reduction of costs as a consequence of a successful intervention.

Overall, the societal costs of patients suffering from ADHD were high. This particularly applied with re- spect to mental health care costs. The assessment of health status showed considerable impairment in the psychological and social functioning of ADHD patients. Furthermore, ADHD patients seem to place an economic burden on the family. Mothers of ADHD patients appear to be confronted with higher direct costs for mental health care, higher indirect costs and more impairment in performing paid and unpaid jobs.

Acknowledgement The study was financed by an unrestricted grant of Eli Lilly Netherlands BV.

\section{References}

1. APA (1994) Diagnostic and statistical manual of mental disorders. DSM-IV, 4th edn. American Psychiatric Association, Washington, DC

2. Birnbaum HG, Kessler RC, Lowe SW, et al. (2005) Costs of attention deficithyperactivity disorder (ADHD) in the US: excess costs of persons with ADHD and their family members in 2000. Curr Med Res Opin 21(2):195-206

3. Dam QDv, Spijker Avt, Arends LR, et al. (1998) Costeffectiveness of psychotherapy: a feasiblity study (in Dutch). J Pychotherapy 24(1):5-22

4. De Ridder A, De Graeve D (2006) Healthcare use, social burden and costs of children with and without ADHD in Flanders, Belgium. Clin Drug Invest 26(2):75-90

5. Escobar R, Soutullo CA, Hervas A, et al. (2005) Worse quality of life for children with newly diagnosed attention-deficit/hyperactivity disorder, compared with asthmatic and healthy children. Pediatrics 116(3):e364-e369

6. Faraone SV (2003) Report from the 4th international meeting of the attention deficit hyperactivity disorder molecular genetics network. Am J Med Genet B Neuropsychiatr Genet 121(1):55-59

7. Guevara J, Lozano P, Wickizer T, et al. (2001) Utilisation and cost of health care services for children with attention-deficit/hyperactivity disorder. Pediatrics 108(1):71-78

8. Hakkaart- van Roijen L (2002) Manual Trimbos/iMTA questionnaire for costs associated with psychaitric illness (in Dutch). Institute for Medical Technology Assessment, Rotterdam

9. Harlow SD, Linet MS (1989) Agreement between questionnaire data and medical records. The evidence for accuracy of recall. Am J Epidemiol 129(2):233248
10. Heliovaara M, Aromaa A, Klaukka T, et al. (1993) Reliability and validity of interview data on chronic diseases. The Mini-Finland Health Survey. J Clin Epidemiol 46(2):181-191

11. Klassen AF, Miller A, Fine S (2004) Health-related quality of life in children and adolescents who have a diagnosis of attention-deficit/hyperactivity disorder. Pediatrics 114(5):e541-e547

12. Koopmanschap MA, Rutten FF (1996) A practical guide for calculating indirect costs of disease. Pharmacoeconomics 10(5):460-466

13. Landgraf J, Abetz L, Ware J (1996) The CHQ's user's manual. The Health Institute, New England Medical Center, Boston

14. Landgraf JM, Maunsell E, Speechley $\mathrm{KN}$, et al. (1998) Canadian-French, German and UK versions of the Child Health Questionnaire: methodology and preliminary item scaling results. Qual Life Res 7(5):433-445

15. Leibson CL, Katusic SK, Barbaresi WJ, et al. (2001) Use and costs of medical care for children and adolescents with and without attention-deficit/hyperactivity disorder. JAMA 285(1):60-66

16. Lesesne CA, Visser SN, White CP (2003) Attention-deficit/hyperactivity disorder in school-aged children: association with maternal mental health and use of health care resources. Pediatrics 111(5 Part 2):1232-1237

17. Linet MS, Harlow SD, McLaughlin JK, et al. (1989) A comparison of interview data and medical records for previous medical conditions and surgery. J Clin Epidemiol 42(12):1207-1213

18. Mannuzza S, Klein RG, Bessler A, et al. (1998) Adult psychiatric status of hyperactive boys grown up. Am J Psychiatry 155(4):493-498
19. Matza LS, Secnik K, Mannix S, et al. (2005) Parent-proxy EQ-5D ratings of children with attention-deficit hyperactivity disorder in the US and the UK. Pharmacoeconomics 23(8):777-790

20. Oostenbrink JB, Al MJ, Rutten-van Molken MP (2003) Methods to analyse cost data of patients who withdraw in a clinical trial setting. Pharmacoeconomics 21(15):1103-1112

21. Oostenbrink JB, Bouwmans CAM, Koopmanschap MA, et al. (2004) Manual for costing, methods and reference unit costs for economic evaluation in health care (in Dutch). College voor zorgverzekeringen

22. Parr JR, Ward A, Inman S (2003) Current practice in the management of Attention Deficit Disorder with Hyperactivity (ADHD). Child Care Health Dev 29(3):215-218

23. Raat H, Botterweck AM, Landgraf JM, et al. (2005) Reliability and validity of the short form of the child health questionnaire for parents (CHQ-PF28) in large random school based and general population samples. J Epidemiol Community Health 59(1):75-82

24. van Roijen L, Essink-Bot ML, Koopmanschap MA, et al. (1996) Labor and health status in economic evaluation of health care. The Health and Labor Questionnaire. Int J Technol Assess Health Care 12(3):405-415

25. Sayal K, Taylor E, Beecham J (2003) Parental perception of problems and mental health service use for hyperactivity. J Am Acad Child Adolesc Psychiatry 42(12):1410-1414

26. Swensen AR, Birnbaum HG, Secnik K, et al. (2003) Attention-deficit/hyperactivity disorder: increased costs for patients and their families. J Am Acad Child Adolesc Psychiatry 42(12):14151423 
27. Wulffraat N, van der Net JJ, Ruperto N, et al. (2001) The Dutch version of the Childhood Health Assessment Questionnaire (CHAQ) and the Child Health Questionnaire (CHQ). Clin Exp Rheumatol 19(4 Suppl 23):S111-S115
28. Zwirs BW, Burger H, Schulpen TW, et al. (2007) Prevalence of psychiatric disorders among children of different ethnic origin. J Abnorm Child Psychol [Epub ahead of print] 